\section{(A) Check for updates}

Cite this: Dalton Trans., 2021, 50 11610

Received 25th June 2021, Accepted 30th July 2021

DOI: $10.1039 / \mathrm{d} 1 \mathrm{dt} 02123 \mathrm{k}$ rsc.li/dalton

\title{
Easy and fast in situ functionalization of exfoliated 2D black phosphorus with gold nanoparticles $\uparrow$
}

\author{
Salvatore Moschetto, (iD *a Andrea lenco, (iD ${ }^{\mathrm{b}}$ Gabriele Manca, (iD) *b \\ Manuel Serrano-Ruiz, (iD b Maurizio Peruzzini, (D) b Alessio Mezzi, iD c \\ Marco Brucale, (D) a Margherita Bolognesi (D) *a and Stefano Toffanin (D) a
}

\begin{abstract}
Heterostructures of single- and few-layer black phosphorus (2D bP) functionalized with gold nanoparticles (Au NPs) have been recently reported in the literature, exploiting their intriguing properties and biocompatibility for catalytic, therapeutical and diagnostic applications. However, a deeper insight on the structural and electronic properties at the interface of the 2D bP/Au NP heterostructure is still lacking. In this work, 2D bP is functionalized with Au nanoparticles (NPs) through in situ deposition-precipitation heterogeneous reaction. The smallest realized Au NPs have a diameter around $10 \mathrm{~nm}$ as revealed by atomic-force and scanning electron microscopy, and are partially positively charged as revealed by $X$-ray Photoelectron Spectroscopy (XPS). XPS, UV-vis and Raman spectroscopy, supported by density functional theory (DFT) calculations, confirmed that while the structural and electronic properties of 2D bP are overall preserved, a soft-pairing between $\mathrm{P}$ atoms at the surface of 2D bP and Au atoms at the surface of Au NPs occurs, leading to a partial charge transfer at the 2D bP/Au interface, with a positive charge being localized on the Au atoms directly bonded to 2D bP. DFT calculations also predicted a band gap lowering, by $0.8 \mathrm{eV}$, for phosphorene functionalized with a tetranuclear Au cluster. Larger effects are expected as the Au cluster nuclearity (and coverage) increases.
\end{abstract}

\section{Introduction}

Single- and few-layer black phosphorus (2D bP) is a promising novel $2 \mathrm{D}$ material that can be easily obtained by either micromechanical cleavage (Scotch tape $\operatorname{method}^{1,2}$ ) or liquid exfoliation $^{3-10}$ of bulk bP, the most stable allotrope of elemental phosphorus at room temperature. ${ }^{11}$ Since its discovery, this new P-based 2D material has attracted enormous interest. In fact, it is endowed with a direct and tunable band gap ranging from $0.3 \mathrm{eV}$ (bulk) up to $2.2 \mathrm{eV}$ (monolayer) and a high carrier mobility (up to $6000 \mathrm{~cm}^{2} \mathrm{~V}^{-1} \mathrm{~s}^{-1}$ ), ${ }^{12}$ which prompted its application towards the production of nanoelectronic devices, such as field effect transistors (FETs). ${ }^{13-15}$ In any case, the material has to be protected in order to prevent its degradation toward oxygen and atmospheric moisture,$^{16-18}$ although in recent

\footnotetext{
${ }^{a}$ National Research Council - Institute for the Study of Nanostructured Materials (CNR-ISMN), Via P. Gobetti, 101, 40129 Bologna, Italy.

E-mail:salvatore.moschetto@ismn.cnr.it,margherita.bolognesi@cnr.it ${ }^{b}$ National Research Council - Institute for the Chemistry of OrganoMetallic Compounds (CNR-ICCOM), Via Madonna del Piano 10, 50019 Sesto Fiorentino, Italy.E-mail: gabriele.manca@iccom.cnr.it

${ }^{c}$ National Research Council - Institute for the Study of Nanostructured Materials (CNR-ISMN), Via salaria km 29.3, 00015 Monterotondo Stazione (Rome), Italy

$\dagger$ Electronic supplementary information (ESI) available. See DOI: 10.1039/ d1dt02123k
}

years also some oxidized forms of $2 \mathrm{D}$ bP have attracted attention on them. ${ }^{19}$

Additional intriguing properties can be obtained through the deposition of metal atoms, molecular fragments or nanoparticles on the surface, which may cause interesting structural and electronic variations. ${ }^{20}$ A systematic study through first-principles calculations on the binding energy, geometry, magnetic moment, and electronic structure of several metal adatoms adsorbed on 2D bP has been carried out, predicting that the immobilization of transition metal atoms on the surface of $2 \mathrm{D} \mathrm{bP}$ is feasible, preserving its structural integrity. ${ }^{21-28}$ More recently, a detailed guideline has been provided for an efficient covalent functionalization of the exfoliated bP with transition metal fragments in different coordination modes taking into account all the possible electronic/ steric features. ${ }^{29}$ An alternative way for the achievement of covalent functionalization could follow from the selective transfer of chalcogenide atoms $(\mathrm{O}, \mathrm{S})$ from suitable sources to the $2 \mathrm{D}$ bP material, in order to anchor the metal complexes and/or nanoparticles. ${ }^{30}$

On the other hand, many experimental studies report on the functionalization of 2D bP with metal nanoparticles (NPs) for a variety of applications including energy generation ${ }^{31}$ and conversion, $^{32}$ sensing $^{33}$ or catalysis. ${ }^{34}$ Much of the experimental work on heterostructures made of 2D bP and NPs deals 
with gold Au NPs generally addressing the biocompatibility of such systems, ${ }^{35}$ together with the properties of singlet oxygen generation ability and localized surface plasmon resonance (LSPR), for diagnostic or therapeutic ${ }^{36}$ or sensing applications. $^{37,38}$ Other works deal with the synthesis of 2D $\mathrm{bP} / \mathrm{Au} \mathrm{NP}$ heterostructures and their catalytic applications. ${ }^{39}$ The efficiency of the system has been attributed to the coupling of the intrinsic catalytic activity of both $2 \mathrm{D} \mathrm{bP}^{40}$ and $\mathrm{Au}$ NPs and to the prevented aggregation of NPs (typically resulting in inhibited catalytic performance), thus a synergic effect can be modulated through an accurate engineering of the heterostructure. ${ }^{39,41}$

However, in all the experimental works dealing with the decoration of $2 \mathrm{D}$ bP with Au NPs very little attention has been devoted to the detailed analysis of the structural and electronic properties at the interface of such heterostructure. In particular, the size control and the growth process of the metal clusters on top of the 2D bP surface is a key step for their application in different technological fields. ${ }^{42-44}$

This work aims at studying 2D bP functionalized in situ with Au NPs through a deposition-precipitation solid-liquid heterogeneous reaction starting from an $\mathrm{Au}(\mathrm{I})$ precursor, which upon reduction reaction may form small nanoparticles. In fact, it is known that a smaller size of Au NPs enhances their catalytic properties due to the larger surface area. ${ }^{45,46}$ In this view, we looked throughout in situ functionalization to obtain $\mathrm{nm}$-sized NPs (diameter of $\sim 10 \mathrm{~nm}$ ) and to eliminate any stabilizing agent that could interfere with the interaction with $2 \mathrm{D}$ bP. Moreover, the in situ functionalization provides a strong intimacy of contact between 2D bP and the metal-precursor, leading well-distributed and homogenous morphology at the nanoscale, which is optimal for investigating the $2 \mathrm{D} \mathrm{bP} / \mathrm{Au}$ interface. Spectroscopic (UV-vis absorption spectroscopy, X-ray photoelectron Spectroscopy, or XPS) and morphological characterization (atomic force microscopy, or AFM), supported by density functional theory (DFT) calculations, are herein reported to provide a deeper understanding on the cross-correlated electronic, structural and morphological characteristics of the $2 \mathrm{D} \mathrm{bP} / \mathrm{Au}$ NPs heterostructure.

\section{Experimental}

\section{Materials}

Bulk bP was synthesized following Lange's procedure. ${ }^{47}$ Chloro (dimethylsulfide)gold(I), [( $\left.\left.\mathrm{Me}_{2} \mathrm{~S}\right) \mathrm{AuCl}\right]$, and solvents (isopropanol, acetone, anhydrous chloroform) were purchased from Sigma-Aldrich. $\mathrm{Si} / \mathrm{SiO}_{2}$ (with thermally grown $\mathrm{SiO}_{2}$, thickness $300 \mathrm{~nm}$ ) and quartz substrates were washed with two cycles of acetone and two of isopropanol in ultrasonic bath before use.

\section{Samples preparation}

We employed the micromechanical cleavage method with blue tape to exfoliate bulk bP to obtain few layers of 2D bP onto Si/ $\mathrm{SiO}_{2}$ substrates or quartz. The substrates resulted covered on $\approx 4 \%$ of their total area by $2 \mathrm{D}$ bP flakes with mean lateral size of $1 \mu \mathrm{m}$ and thickness ranging from $50 \mathrm{~nm}$ down to few $\mathrm{nm} .^{48}$ Quartz/2D bP and $\mathrm{Si} / \mathrm{SiO}_{2} / 2 \mathrm{D}$ bP samples were functionalized with gold nanoclusters by dipping them in a $10^{-3} \mathrm{M}$ solution of $\left[\left(\mathrm{Me}_{2} \mathrm{~S}\right) \mathrm{AuCl}\right]$ in anhydrous $\mathrm{CHCl}_{3}$. The functionalization was carried out under an inert atmosphere of $\mathrm{N}_{2}$, in the glove box, in the dark and at ambient temperature. Immersion times of 3, 18 and 72 hours were tested. Samples were finally gently rinsed with degassed and anhydrous chloroform.

\section{Samples characterization}

Micro-Raman. Micro-Raman spectra were recorded by using a Renishaw micro-Raman 1000 system exciting with a laser diode at $488 \mathrm{~nm}$. The laser beam was focused through a $50 \times$ objective.

UV-Vis absorption. The absorption spectra of quartz/2D bP substrates functionalized with Au NPs were recorded with a JASCO V-550 spectrophotometer.

Atomic force microscopy (AFM). Atomic force microscopy images were recorded on a Multimode 8 microscope equipped with a Nanoscope $\mathrm{V}$ controller and a type JV piezoelectric scanner (Bruker, USA). Samples were scanned in PeakForce mode with SNL-A probes (Bruker, USA) under dry nitrogen. Background correction and grain size analysis were performed with Gwyddion 2.48 (http://gwyddion.net/). ${ }^{49}$

Scanning electron microscopy (SEM). The morphology of the $\mathrm{Au}$ nanoclusters of the $2 \mathrm{D} \mathrm{bP/Au} \mathrm{NP}$ heterostructure on $\mathrm{Si}$ / $\mathrm{SiO}_{2}$ substrates was also investigated with: (i) a scanning electron microscope FEG LEO 1530, working with an acceleration voltage of $5 \mathrm{kV}$, equipped with energy dispersive X-ray spectrometer (EDS), Oxford SDD Inca system; and a scanning electron microscope (SEM) Zeiss EVO LS 10 working with an acceleration voltage of $20 \mathrm{kV}$ equipped with EDS Bruker SDD Quantax system. FEG-SEM micrographs were collected after depositing the solid samples on a stub provided with a double sided adhesive conductive carbon tape and coated by evaporation with $10 \mathrm{~nm}$ of carbon. We performed the elemental mapping on the sample by EDS supported by the above described SEM, LEO 1530 FEG and Zeiss EVO LS 10.

X-ray photoelectron spectroscopy (XPS). XPS measurements were carried out by using a ESCALAB 250Xi spectrometer (Thermo Fisher Scientific - UK) equipped with a monochromatic $\mathrm{Al} \mathrm{K \alpha}$ source and six-channeltron as detection system. The apparatus works in a UHV system, where the base pressure of the analysis chamber during the experiments was approximately $1 \times 10^{-10}$ mbar. The analyzed area of the sample was varied in the range of 100-900 $\mu \mathrm{m}$, by means an electromagnetic lens system. The spectra were collected operating at constant pass energy $\mathrm{CAE}=50 \mathrm{eV}$. In order to minimize bP oxidation, the sample was manipulated and mounted on the holder for XPS analysis under $\mathrm{N}_{2}$ atmosphere in a glove box. The BE scale was calibrated positioning the $\mathrm{C}$ 1s peak from adventitious carbon at $\mathrm{BE}=285.0 \mathrm{eV}$. The accuracy of the measurements was $\pm 0.1 \mathrm{eV}$. The spectroscopic data were acquired and processed by using the Avantage v.5 software.

Computational methods. All the calculations were performed within CRYSTAL17 software package ${ }^{50}$ at B3LYP-DFT 
level of theory ${ }^{51}$ by considering a 32 phosphorus atom supercell. All the structures have been optimized as minima for both the atomic positions and the lattice parameters. The TZVP basis set was used for the phosphorus atoms while pseudo-potential was employed for the inner electrons of the $\mathrm{Au}$ center(s). ${ }^{52,53} \mathrm{~A}$ list of all the optimized structural and energy parameters is available in the ESI. $\dagger^{29}$

\section{Results and discussion}

For the 2D bP functionalization with Au NPs, we choose the direct addition of an $\mathrm{Au}(\mathrm{I})$ complex solution on the surface of bP. The precursor complex, [( $\left.\left.\mathrm{Me}_{2} \mathrm{~S}\right) \mathrm{AuCl}\right]$, with one $\mathrm{Cl}^{-}$ligand and a labile dimethylsulfide ligand, easily reduces to gold NPs when added to a suspension of few layers $2 \mathrm{D}$ bP in $\mathrm{CHCl}_{3}$. We expressly avoided to use Au NPs stabilized with organic surfactants to avoid covering of the 2D bP surface by the surfactant through non-covalent interactions, ${ }^{48}$ as it occurs also for graphene, ${ }^{5,55}$ which could hinder the P-Au interaction. The solid-liquid heterogeneous reaction was carried out for a range of reaction times (see Experimental section), in order to find the best compromise between a good substrate coverage and minimum size of the NPs.

\section{Spectroscopic characterization}

Raman spectroscopy was carried out on pristine 2D bP and on Au-functionalized samples (Fig. 1). Both spectra show the three typical Raman peaks of bP at 360, 435 and $460 \mathrm{~cm}^{-1}$, attributed to the $\mathrm{A}_{\mathrm{g}}^{1}, \mathrm{~B}_{2 \mathrm{~g}}$, and $\mathrm{A}_{\mathrm{g}}^{2}$ vibrational modes, respectively. ${ }^{56-58}$ This confirms that the orthorhombic crystalline structure of $2 \mathrm{D}$ bP is maintained after Au-functionalization, at least in the atomic-thin layers of $2 \mathrm{D}$ bP under the functionalized surface. In fact, as reported in the literature, covalent surface functionalization of $2 \mathrm{D}$ bP causes only a slight weakening of all bP Raman features (in particular of the $\mathrm{A}^{1}$ g mode) due to the partial disruption of intra-layer phosphorus bonding. ${ }^{59}$

UV-vis spectroscopy on pristine $2 \mathrm{D}$ bP and Au-functionalized samples on quartz (incubation time 3 h, Fig. 2) resulted in broad and featureless absorption spectra for both samples,

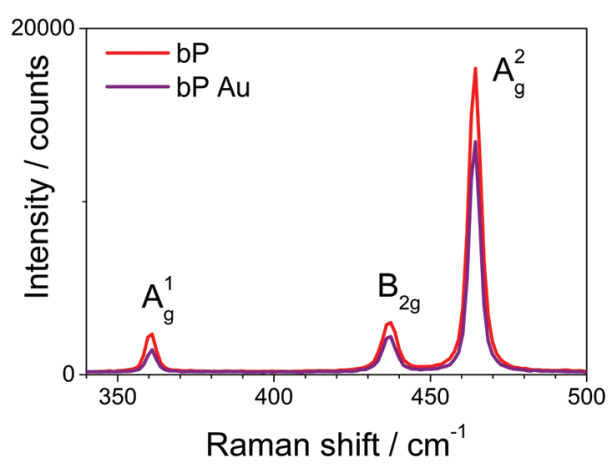

Fig. 1 Raman spectra of pristine 2D bP (red) and Au-functionalized 2D $\mathrm{bP}$ (purple) on $\mathrm{Si} / \mathrm{SiO}_{2}$ substrates.

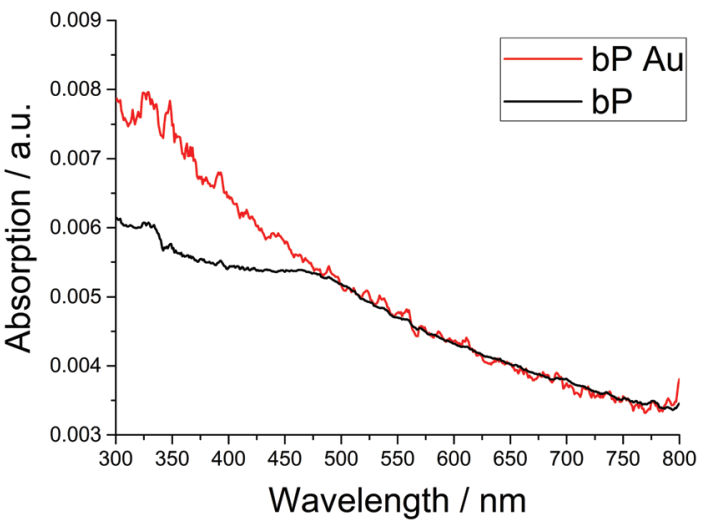

Fig. 2 Absorption spectra of mechanically exfoliated pristine bP (black) and bP functionalized with gold NPs (red) on quartz substrates.

typical of bP. The expected peak showing plasmonic effects, (at about $520 \mathrm{~nm}$, typical of Au NPs with size $<10 \mathrm{~nm}$ as described in the following morphological characterization), ${ }^{32}$ is not present. This may be either ascribed to: (i) a low coverage of $\mathrm{Au}$ nanoclusters (ii) quenching by $2 \mathrm{D} \mathrm{bP}$, or, (iii) a combination of both factors. These assumptions are supported by literature, where it is supposed that the realization of $2 \mathrm{D}$ bPanchored $\mathrm{Au}$ nanoclusters follows a kinetically controlled process, ${ }^{60}$ and quenching effects occur also for graphene and other 2D materials. ${ }^{61,62}$ Forming $\mathrm{Au}$ nanoclusters on the surface of $2 \mathrm{D}$ bP under experimental conditions featuring a low ratio between the $\mathrm{Au}$ precursor and the $2 \mathrm{D}$ bP (down to $2.5 \%)$ might enhance the collision probability between $\mathrm{Au}(\mathrm{I})$ atoms from the precursor and $\mathrm{P}$ centers from $2 \mathrm{D}$ bP, enabling an easier study of the heterostructure due to the large fraction of $\mathrm{Au}-\mathrm{P}$ binding sites. However, we cannot exclude a-priori that the increase of the absorption spectrum of the $2 \mathrm{D} \mathrm{bP/Au}$ functionalized sample in the $400-475 \mathrm{~nm}$ spectral range is due to scattering effects.

As a surface-sensitive mass spectroscopic technique, XPS was used to shed light on the elemental composition, the stoichiometry, the chemical and electronic state of the elements present in our heterostructure systems. The XPS analysis of bulk bP, from which the 2D bP was exfoliated, evidenced the presence of elemental phosphorus characterized by the typical spin-orbit doublet $\mathrm{P} 2 \mathrm{p}_{3 / 2}-\mathrm{P} 2 \mathrm{p}_{1 / 2}$, separated by $0.9 \mathrm{eV}$, and positioned at $\mathrm{BE}=130.0 \mathrm{eV}$ (see Fig. S1 and Table S1 in the ESI†).

In the case of Au-functionalized sample (incubation time $3 \mathrm{~h}$ ), the XPS measurements were performed on different areas of the sample. Since the lateral resolution of the XPS equipment is approximately of $20 \mu \mathrm{m}$, it was not possible to focus the X-ray beam on a single $2 \mathrm{D}$ bP flake, which, on average, presents typically shorter lateral dimension (see the optical microscopy images in Fig. S2 in the ESI $\dagger$ ). However, it was possible to distinguish between two categories of regions, depending on their elemental composition, namely regions "A" and "B" respectively (see Table 1). In regions A, signals from $\mathrm{Au}$ and $\mathrm{P}$ atoms were found, while in regions $\mathrm{B}$, signals 
Table 1 Binding energy (BE) and atomic concentration (atomic \%), acquired in two different areas of the $2 \mathrm{D}$ bP flakes on the $\mathrm{Si} / \mathrm{SiO}_{2}$ substrate functionalized with Au NPs

\begin{tabular}{|c|c|c|c|}
\hline Signal & Peak BE $(\mathrm{eV})$ & Atomic \% & Bond \\
\hline \multicolumn{4}{|l|}{ Region A } \\
\hline Au $4 f_{7 / 2}$ & 84.3 & 0.3 & $\mathrm{Au}^{+}$ \\
\hline $\mathrm{C} 1 \mathrm{~s}-1$ & 285.0 & 9.9 & $\mathrm{C}-\mathrm{C}$ \\
\hline $\mathrm{C} 1 \mathrm{~s}-2$ & 287.3 & 1.6 & $\mathrm{C}-\mathrm{O}$ \\
\hline O1s & 533.0 & 54.4 & $\mathrm{SiO}_{2}$ \\
\hline $\mathrm{P} 2 \mathrm{p}$ & 129.9 & 2.3 & $\mathrm{P}$ \\
\hline $\operatorname{Si} 2 \mathrm{p}$ & 103.6 & 31.6 & $\mathrm{SiO}_{2}$ \\
\hline \multicolumn{4}{|l|}{ Region B } \\
\hline $\mathrm{Au} 4 f_{7 / 2}-1$ & 85.2 & 0.23 & $\mathrm{Au}^{+}$ \\
\hline $\mathrm{Au} 4 \mathrm{f}_{7 / 2}-2$ & 87.7 & 0.21 & $\mathrm{Au}^{3+}$ \\
\hline $\mathrm{C} 1 \mathrm{~s}-1$ & 285.0 & 11.5 & $\mathrm{C}-\mathrm{C}$ \\
\hline $\mathrm{C} 1 \mathrm{~s}-2$ & 286.7 & 2.7 & $\mathrm{C}-\mathrm{O}$ \\
\hline $\mathrm{C} 1 \mathrm{~s}-3$ & 289.1 & 1.0 & $-\mathrm{COOH}$ \\
\hline $\mathrm{Cl} 2 \mathrm{p}_{3 / 2}$ & 199.2 & 1.2 & $\mathrm{Cl}^{-}$ \\
\hline N1s & 400.9 & 0.9 & Ammonium \\
\hline O1s & 533.2 & 48.4 & $\mathrm{SiO}_{2}$ \\
\hline $\operatorname{Si} 2 p-1$ & 103.9 & 32.7 & $\mathrm{SiO}_{2}$ \\
\hline Si2p -2 & 101.5 & 1.2 & $\mathrm{Si}-\mathrm{O}$ \\
\hline
\end{tabular}

from $\mathrm{Au}$ and $\mathrm{Cl}$ atoms were found. Both kind of regions were characterized also by signals from $\mathrm{Si}$ and $\mathrm{O}$ atoms, deriving from the silicon/silicon oxide substrate, and $\mathrm{C}$ atoms, ascribed to surface contamination with organic impurities or scotch tape residuals from the mechanical exfoliation process.

The $\mathrm{P} 2 \mathrm{p}$ peak detected in the A regions was positioned at $\mathrm{BE}=130.0 \mathrm{eV}$, characteristic for elemental $\mathrm{P}$, as it was found in the pristine bP reference sample. Therefore, it can be attributed to $2 \mathrm{D} \mathrm{bP}^{63}$ (Fig. 3). In addition, the $\mathrm{Au} 4 \mathrm{f}_{7 / 2}$ peak was positioned at $\mathrm{BE}=84.4 \div 84.8 \mathrm{eV}$ and it was assigned to $\mathrm{Au}$ arranged in NPs. As it is well known, the typical $A u 4 f_{7 / 2}$ peak of bulk gold $\left(\mathrm{Au}^{0}\right)$ is positioned at $\mathrm{BE}=84.0 \mathrm{eV}$. Thus, the observed chemical shift strongly suggests an electronic interaction occurring between $\mathrm{Au}$ atoms and the 2D bP surface.
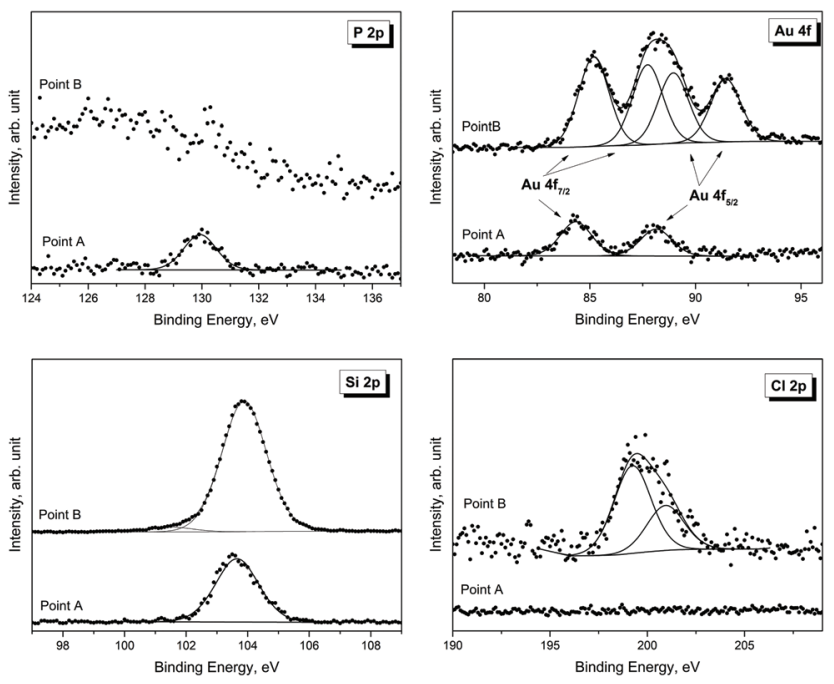

Fig. 3 XPS spectra, acquired in regions $A$ and $B$ of samples of 2D bP flakes functionalized with $\mathrm{Au} \mathrm{NPs}$ on a $\mathrm{Si} / \mathrm{SiO}_{2}$ substrate.
In the $\mathrm{B}$ regions, where $2 \mathrm{D}$ bP was absent (no $\mathrm{P} 2 \mathrm{p}$ signal was detected), the $\mathrm{Au}$ if signal showed two doublets $A u 4 \mathrm{f}_{7 / 2^{-}}$ Au4f $\mathrm{f}_{5 / 2}$, assigned to two different electronic configurations of $\mathrm{Au}$ centers (i.e. $\mathrm{Au}^{+}$and $\left.\mathrm{Au}^{3+}\right) .{ }^{64}$ In the same point, also the $\mathrm{Cl}$ $2 \mathrm{p}$ signal was detected at peak position with $\mathrm{BE}=199.2 \mathrm{eV}$. This value is characteristic for $\mathrm{Cl}^{-}$, most probably due to the presence of $\mathrm{Cl}^{-}$counteranions necessary to electronically stabilize $\mathrm{Au}^{+}$and $\mathrm{Au}^{3+}$ atoms on the NPs surface in the absence of $2 \mathrm{D}$ bP.

\section{Morphological characterization}

Topographical AFM investigation of bare 2D bP before functionalization confirmed the expected planar and relatively featureless morphology (Fig. 4a) with occasional sharp steps of several $\AA$ (the smallest measuring $6.6 \pm 1.0 \AA$, see Fig. 4e), suggesting the good quality of the $2 \mathrm{D}$ bP flakes prepared through the micromechanical exfoliation method. Controlled oxidation of $2 \mathrm{D}$ bP in air led initially to the formation on the surface of raised features similar to bubbles, which progressively grew in size upon further oxidation (see Fig. S3†). The oxidation bubbles were clearly discernible from the undamaged substrate via the Peak Force phase contrast, which attests a substantial difference between the nanomechanical characteristics of pristine $\mathrm{bP}$ and oxidized zones.

Fig. 4b, $\mathrm{c}$ and d show the surface morphology of $2 \mathrm{D}$ bP flakes incubated with $\left[\left(\mathrm{CH}_{3}\right)_{2} \mathrm{~S}\right] \mathrm{AuCl}$ for respectively 3,18 and $72 \mathrm{~h}$. In all cases, the incubation process did not induce appreciable oxidation damage on bP (as it is observed instead for $2 \mathrm{D}$ bP in air, see Fig. $\mathrm{S} 3 \dagger$ ) thus the Au NPs coverage is preserved on the $2 \mathrm{D}$ bP (see for comparison bare $2 \mathrm{D}$ bP in Fig. S2b in the ESI $\dagger$ ). The sample incubated for $3 \mathrm{~h}$ is largely decorated by isolated spherical Au NPs with an average size of $=10.2 \pm 3.5 \mathrm{~nm}$ (Fig. 4f). Longer incubation times led to the formation of larger $\mathrm{Au}$ NPs clusters in addition to those observed on the sample incubated for $3 \mathrm{~h}$. At $18 \mathrm{~h}$, the average diameter of these larger aggregates is $27.0 \pm 10.4 \mathrm{~nm}$ (Fig. $4 \mathrm{~g}$ ), while at $72 \mathrm{~h}$ the distribution further broadens to include aggregates up to $100 \mathrm{~nm}$ in diameter (Fig. 4h). All the larger Au clusters appear to be constituted by smaller grains having a diameter of $\sim 10 \mathrm{~nm}$ (see insets of Fig. $4 \mathrm{c}$ and d).

Considering all these observations, we can assess that the chosen functionalization procedure is effective in producing nm-sized NPs with a high affinity towards the 2D bP surface, in perfect accordance with the optical, Raman and XPS spectroscopic characterizations.

The morphology and size distribution of $\mathrm{Au}$ nanoclusters on the 2D bP surface were further confirmed by SEM results (Fig. S4†).

\section{Computational studies}

In order to shed light on the absorption sites, energy and bonding nature of the $\mathrm{Au}$ nanoclusters on 2D bP evidenced by spectroscopic and microscopic investigations, a detailed Density Functional Theory (DFT) computational study was carried out. The optimization of the single layer of the black phosphorus (monolayer 2D bP, or phosphorene) reproduced 

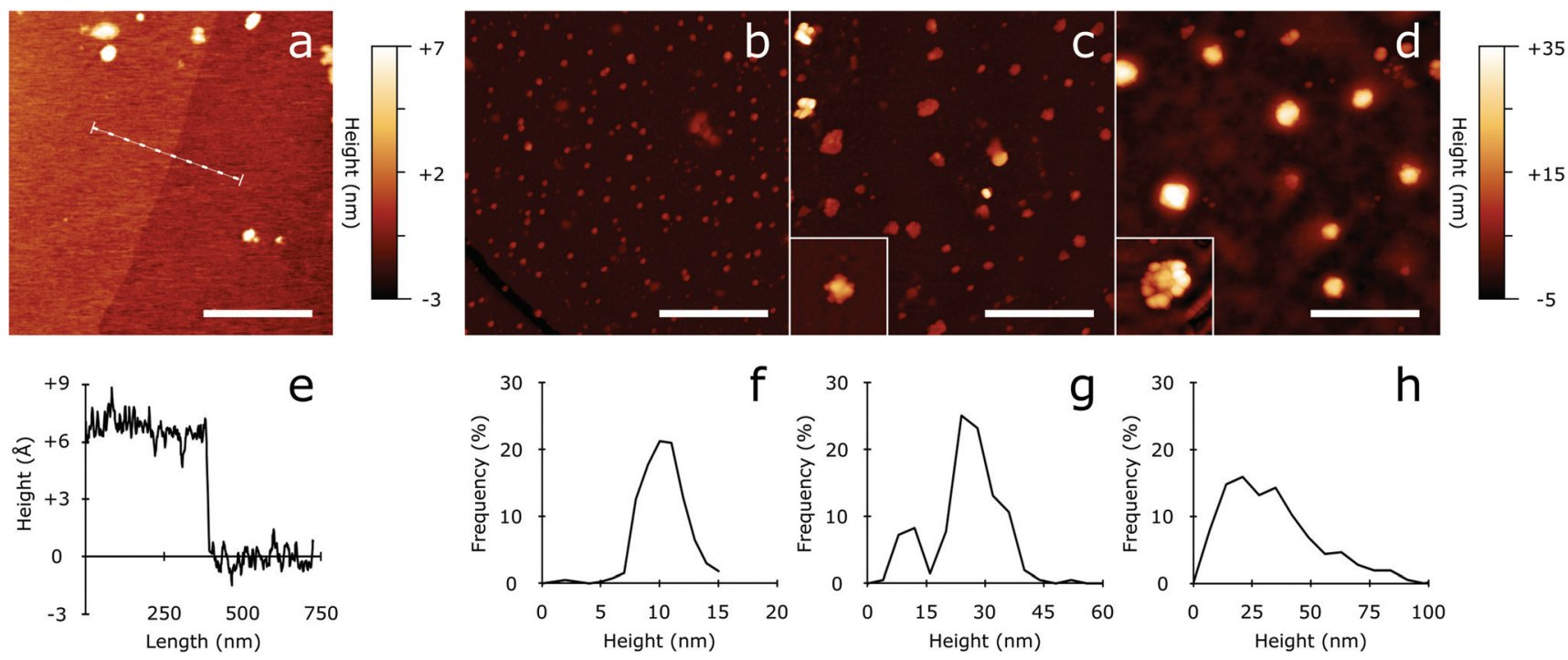

Fig. 4 (a-d): representative AFM micrographs of bP flakes immediately after mechanical cleavage (a) and after incubation with [(Me $\left.{ }_{2} \mathrm{~S}\right) \mathrm{AuCl}$ for $3 \mathrm{~h}$ (b), $18 \mathrm{~h}$ (c) and $72 \mathrm{~h}$ (d). All scale bars are $500 \mathrm{~nm}$. Insets of panel (c) and (d) show $4 \times$ magnifications of the larger Au clusters found on the corresponding sample. (e): height profile of a typical step found on pristine bP measured along the dashed line shown in panel (a). ( $f-h)$ : maximum vertical

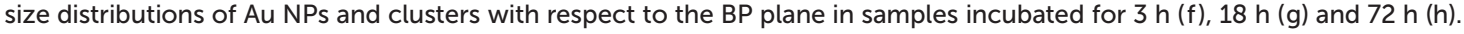

the experimental structure with two in-plane short P-P distances of $2.24 \AA$ and one longer out of plane of $2.29 \AA$ with a calculated channel width of $3.63 \AA$ A.

The absorption of a single $\mathrm{Au}(0)$ atom on the phosphorene surface revealed a non-symmetric interaction between $\mathrm{Au}$ and three $\mathrm{P}$ centres, with one shorter $(2.36 \AA)$ and two longer $(2.55 \AA)$ bond lengths, caused by different reciprocal orientations of the lone pairs of $\mathrm{P}$ atoms. ${ }^{29}$ The absorption energy $\left(E_{\text {abs }}\right)$ was calculated as large as $-2.5 \mathrm{eV}$. This $E_{\text {abs }}$ value is somewhat overestimated comparing to the one reported in the literature $(-1.61 \mathrm{eV})$ due to the employment of different hybrid functionals (B3LYP rather than PBE0), whereas from a structural point of view the two results are in perfect agreement. ${ }^{21}$ In any case as shown in Fig. 5, the absorption of the metal atom causes a distortion of the structure, never put in evidence by the precedent investigation where the attention was focused

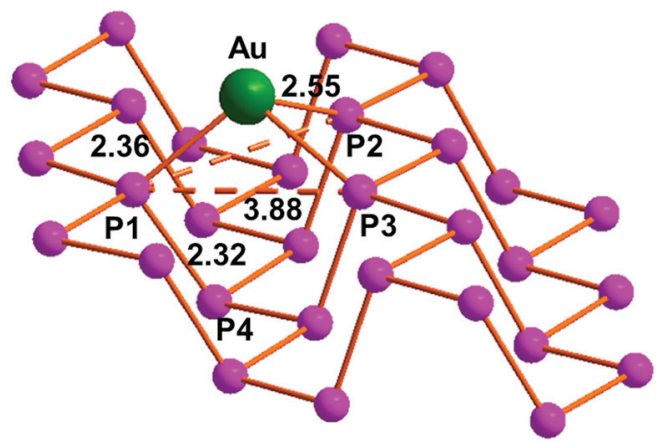

Fig. 5 Optimized structure of the black phosphorus monolayer after the absorption of a single gold atom. only on the out of-plane P-P distance rather than on the width of the channels. In this regards, our calculations revealed channels wider by around $0.25 \AA$ compared to the naked phosphorene (3.63 $)$ ), a trend similar to what already obtained for the predicted functionalization of the phosphorene with $\mathrm{AuCl}$ fragment although the asymmetry in the $\mathrm{Au}-\mathrm{P}$ bond is more pronounced. ${ }^{29}$ Otherwise in our calculations, the variation of the out-of-plane P-P distance (between P1-P4 atoms, Fig. 5) is only $0.03 \AA$ larger compared to the naked phosphorene layer.

The Mulliken analysis revealed a +0.35 charge for the $\mathrm{Au}$ atom without any significant variations between the three involved $\mathrm{P}$ atoms. Recent publications revealed the oxidation of an adatom upon the interaction with the phosphorene surface for the case of a single copper atom, which donates its unpaired electron into the conduction band of the material. ${ }^{65}$ Our calculations highlighted an electronic population for the $\mathrm{Au}$ center of $0.49 \mathrm{e}^{-}$less than the expected value for an $\mathrm{Au}$ atom at 0 oxidation state, suggesting a somewhat degree of electron donation of the metal towards the phosphorene layer.

On this basis, the adsorption on the 2D bP surface of the simplest seed of an $\mathrm{Au}$ nanocluster, namely two bonded $\mathrm{Au}$ atoms (Au1-Au2), was computed. For the Au1-Au2 assembly, two different arrangements on the $2 \mathrm{D}$ bP surface were investigated, as reported in Fig. 6.

The optimization revealed that the most stable conformation is the one shown in Fig. 6b, with $E_{\text {abs }}$ energy of $-2.4 \mathrm{eV}$, more stable by $0.7 \mathrm{eV}$ compared to the conformation parallel to the channel (Fig. 6a). From a structural viewpoint, the Au center in the channel (Au1) is now bonded to a P1 with a bond length of $2.28 \AA$ and to the other $\mathrm{Au}$ center $(\mathrm{Au} 2)$, with a metal-to-metal distance of $2.53 \AA$ and P1-Au1-Au2 angle of $162^{\circ}$. The other two Au1-P bond distances elongate by $0.4 \AA$, 


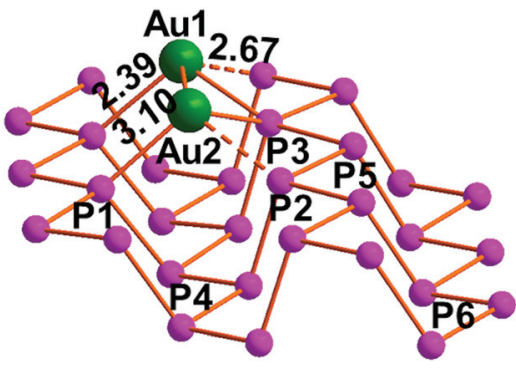

a

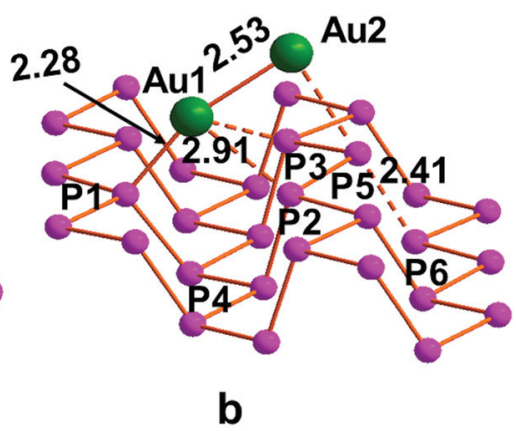

Fig. 6 Optimized different configurations for the $A u_{1}-A u_{2}$ moiety on phosphorene: (a) parallel to the channel and (b) over the channel with a leaning structure (one gold on the channel and bonded to another $\mathrm{Au}$, interacting with a fourth phosphorus of the surface), respectively.

as compared to the single $\mathrm{Au}$ atom on phosphorene, reaching a limit value of $2.91 \AA$. Differently from Au1, the Au2 center only weakly interacts with the surface through a single $\mathrm{P}$ atom being the Au2-P5 distance $2.76 \AA$ A. Such an interaction in any case causes a structural deformation since the P5-P6 bond results elongated up to 2.41 vs. $2.31 \AA$, similarly to the recent chalcogen atom transfer from a molecule of stibine chalcogenide to phosphorene. ${ }^{30}$

The absorption of the $\mathrm{Au}_{2}$ cluster on the phosphorene causes a less pronounced deformation being the ditch larger by only $0.18 \AA$. Concerning the charge distribution, also in this case a charge transfer is observed, especially from the Au1 center, which seems to be partially oxidized being its population $0.36 \mathrm{e}^{-}$less than the atomic $\mathrm{Au}(0)$, while the $\mathrm{Au} 2$ remains very close to the $\mathrm{Au}(0)$ electronic configuration. The effect of the elongation of the P5-P6 bond is due to a somewhat donation of electron population from $\mathrm{Au}$ to the $\mathrm{P}-\mathrm{P} \sigma^{*}$ level being the P5-P6 overlap population halved than the pristine naked phosphorene.

In a scale-up approach, the interaction between a cluster made of four $\mathrm{Au}$ atoms $(\mathrm{Au} 1,2,3,4)$ with the 2D bP surface was computed. Five alternative arrangements of the Au1, Au2, Au3, Au4 moiety were investigated, i.e. along, orthogonal or outside the channels. Some configuration were discharged since causing a partial distortion/disruption of the phosphorene crystallinity, which sharply contrasts with XPS results. The

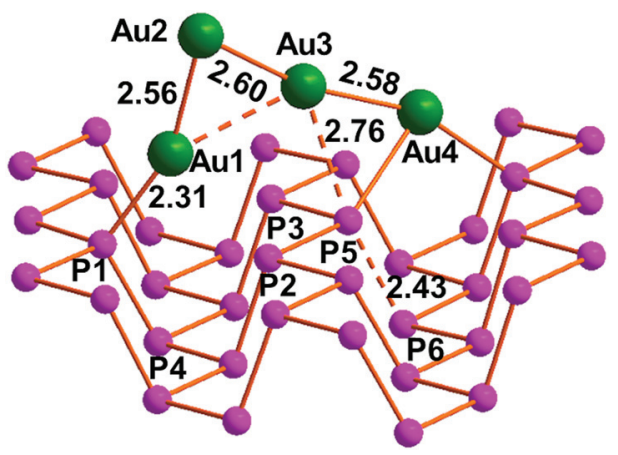

Fig. 7 Optimized structure of phosphorene surface with $\mathrm{Au}_{4}$ cluster. most stable configuration is the one shown in Fig. 7, featuring an adsorption energy as large as $-3.7 \mathrm{eV}$. In this configuration, two $\mathrm{Au}$ atoms (Au1 and Au4) interact with two P ones (P1 and P5, respectively) with a short distance of 2.31 and $2.37 \AA$. The possible interaction of Au1 with other P atoms has to be discarded in view of their long distances (around 3.00 $⿱$ A). While $\mathrm{Au} 2$ is only bounded to its neighboring $\mathrm{Au} 1$ and $\mathrm{Au} 3$ centers, an additional weak interaction is revealed between the gold center $(\mathrm{Au} 3 \mathrm{or} \mathrm{Au} 4)$ and the underlying phosphorene (bond lengths of $2.76 \AA$ and $2.68 \AA$ respectively). Interestingly, $\mathrm{Au} 2$, $\mathrm{Au} 3$ and $\mathrm{Au} 4$ centers attain an almost linear coordination with an angle of $c a \cdot 160^{\circ}$. As occurs for the binuclear Au1-Au2 also in this case an elongation of P5-P6 bond by $c a$. $0.14 \AA$ is observed due to a somewhat degree of electron donation from the Au assembly into the $\sigma^{*}$ level.

From calculations, also a slight positive charge $(+0.2)$ localized on the Au1 and Au4 atoms directly bonded to the P centers of phosphorene was revealed. This, together with the observation of the positive overlap population of the Au-P interactions, is in agreement with XPS measurements, indicating that $\mathrm{Au}$ NPs and nanoclusters present a fraction of $\mathrm{Au}$ atoms (most probably the superficial ones) in an oxidation state higher than 0.

Although the simulated cluster is far from being comparable, in dimensions, with the Au NPs experimentally obtained, the computed system was proven to be a good model for extrapolating energetic and structural information on the experimental one.

From an energetic point of view, simulations confirmed the propensity of $2 \mathrm{D} \mathrm{bP}$ to bind the Au NPs by fixing a fraction of $\mathrm{Au}$ atoms at their periphery through a covalent bond. From a structural viewpoint, simulations also confirmed that the 2D bP crystalline structure is perfectly preserved after the adsorption of Au NPs. Finally, a partial charge transfer between P and $\mathrm{Au}$ atoms is revealed, with a positive charge being localized on the $\mathrm{Au}$ atoms directly bonded to the $2 \mathrm{D} \mathrm{bP}$. This may also explain the quenching of the plasmonic band of the Au NPs in the $2 \mathrm{D} \mathrm{bP/Au} \mathrm{NPs} \mathrm{heterostructure,} \mathrm{as} \mathrm{shown} \mathrm{by} \mathrm{absorption}$ spectroscopy.

The adsorption of gold nanoclusters causes some significant electronic variation in particular concerning the band 
gap. In this respect, the naked phosphorene has an estimated direct band gap of 2.20 eV29 while the more is the metal content in the nanocluster and the smaller becomes the band gap. Such a behavior could be reasonably traced back to the presence of filled $d$ orbitals of the metal at the top of the valence band. In a different way, the conduction band, mainly formed by the $\sigma^{*} \mathrm{P}-\mathrm{P}$ bond, is only slightly influenced in view of limited metal back-donation into empty $\mathrm{P}-\mathrm{P} \sigma^{*}$ bonding. Thus, the band gap becomes progressively smaller as the nuclearity of the particle increases. In this regards, the band gap starting from the $2.20 \mathrm{eV}$ for the naked phosphorene reaches the lowest limit of $1.41 \mathrm{eV}$ for phosphorene functionalized with the tetranuclear gold cluster. The greater is the size of the gold cluster and the smaller becomes the bandgap. The great contribution from the gold atoms at the top of the valence band is mainly attributed to gold-gold bond mediated by a significant contribution from the phosphorus, as shown by the Density of States (DOS) in ESI, Fig. S5 and S6 $\uparrow$ for the material functionalized with $\mathrm{Au}_{2}$ and $\mathrm{Au}_{4}$ moieties, respectively.

\section{Conclusions}

This work aimed at deeply investigating the electronic, structural and morphological properties of a heterostructure formed by $2 \mathrm{D}$ bP and Au NPs. The Au NPs, formed in situ through a solid-liquid heterogeneous reaction, have nanometric size (diameter $\sim 10 \mathrm{~nm}, \sim 27 \mathrm{~nm}$ and $\sim 100 \mathrm{~nm}$ ) as evidenced by AFM, and do not present plasmonic features due to quenching by $2 \mathrm{D} \mathrm{bP}$. In the heterostructure, $2 \mathrm{D}$ bP fully preserves its crystalline structure beneath the interface with $\mathrm{Au}$ NPs, as evidenced by Raman spectroscopy. The formation of the heterostructure involves the soft-pairing/coordination between $\mathrm{P}$ atoms from the surface of $2 \mathrm{D}$ bP to the Au atoms on the surface of the Au NPs. A partial charge transfer at the 2D $\mathrm{bP} / \mathrm{Au}$ interface occurs, with a positive charge being localized on the $\mathrm{Au}$ atoms directly bonded to $2 \mathrm{D} \mathrm{bP}$, as predicted by calculations and confirmed by XPS experiments. Calculations finally predict, for Au-functionalized 2D bP, a band gap lowering which is more pronounced as the nuclearity of the Au clusters increases: i.e. a band gap decrease by $0.8 \mathrm{eV}$ is calculated for phosphorene functionalized with a tetranuclear Au cluster. We expect that for a further increase of the cluster size, up to nanoparticles with $>100$ atoms, the more efficient is the charge transfer, as well as the smaller is the band gap of phosphorene. The results here presented give a theoretical insight and an overall deeper understanding of the interface in the heterostructures based on 2D bP/Au NPs, already widely used for optical and electrochemical sensing ${ }^{66}$ applications, or in plasmonic- or photonic- based biological applications. ${ }^{67}$ On the other hand, the specific heterostructure here designed and studied, obtained through an easy and in situ method leading to a "stabilizer-free" surface and optimal nanostructuring, holds promise for improved performance in specific applications, such as electro-, photo- or photoelectro-chemical catalysis, within others.

\section{Conflicts of interest}

There are no conflicts to declare.

\section{Acknowledgements}

The authors thank Mr. Franco Corticelli (CNR-IMM, Bologna) for the SEM-EDX measurements, and Mr. Vincenzo Ragona (CNR-ISMN, Bologna) and Dr. Federico Prescimone (CNR-ISMN) for the technical support. Thanks are expressed to the European Research Council (ERC) under the European Union's Horizon 2020 research and innovation program (Grant Agreement No. 670173) for funding the project PHOSFUN by an ERC Advanced Grant to M.P.

\section{Notes and references}

1 L. Li, Y. Yu, G. J. Ye, Q. Ge, X. Ou, H. Wu, D. Feng, X. H. Chen and Y. Zhang, Nat. Nanotechnol., 2014, 9, 372377.

2 H. Liu, A. T. Neal, Z. Zhu, Z. Luo, X. Xu, D. Tománek and P. D. Ye, ACS Nano, 2014, 8, 4033-4041.

3 M. Serrano-Ruiz, M. Caporali, A. Ienco, V. Piazza, S. Heun and M. Peruzzini, Adv. Mater. Interfaces, 2016, 3, 1500441.

4 J. Kang, J. D. Wood, S. A. Wells, J.-H. Lee, X. Liu, K.-S. Chen and M. C. Hersam, ACS Nano, 2015, 9, 3596-3604.

5 P. Yasaei, B. Kumar, T. Foroozan, C. Wang, M. Asadi, D. Tuschel, J. E. Indacochea, R. F. Klie and A. SalehiKhojin, Adv. Mater., 2015, 27, 1887-1892.

6 A. H. Woomer, T. W. Farnsworth, J. Hu, R. A. Wells, C. L. Donley and S. C. Warren, ACS Nano, 2015, 9, 88698884.

7 H. Wang, X. Yang, W. Shao, S. Chen, J. Xie, X. Zhang, J. Wang and Y. Xie, J. Am. Chem. Soc., 2015, 137, 1137611382.

8 Z. Guo, H. Zhang, S. Lu, Z. Wang, S. Tang, J. Shao, Z. Sun, H. Xie, H. Wang, X.-F. Yu and P. K. Chu, Adv. Funct. Mater., 2015, 25, 6996-7002.

9 Z. Sun, H. Xie, S. Tang, X.-F. Yu, Z. Guo, J. Shao, H. Zhang, H. Huang, H. Wang and P. K. Chu, Angew. Chem., Int. Ed., 2015, 54, 11526-11530.

10 E. A. Lewis, J. R. Brent, B. Derby, S. J. Haigh and D. J. Lewis, Chem. Commun., 2017, 53, 1445-1458.

11 C. E. Housecroft and A. G. Sharpe, Inorganic Chemistry, Pearson, Prentice Hall, 2nd edn, 2004, p. 392.

12 L. Li, F. Yang, G. J. Ye, Z. Zhang, Z. Zhu, W. Lou, X. Zhou, L. Li, K. Watanabe, T. Taniguchi, K. Chang, Y. Wang, X. H. Chen and Y. Zhang, Nat. Nanotechnol., 2016, 11, 593597.

13 S. Das, W. Zhang, M. Demarteau, A. Hoffmann, M. Dubey and A. Roelofs, Nano Lett., 2014, 14, 5733-5739.

14 V. Tran, R. Soklaski, Y. Liang and L. Yang, Phys. Rev. B: Condens. Matter Mater. Phys., 2014, 89, 235319. 
15 A. Castellanos-Gomez, J. Phys. Chem. Lett., 2015, 6, 42804291.

16 M. Bolognesi, S. Moschetto, M. Trapani, F. Prescimone, C. Ferroni, G. Manca, A. Ienco, S. Borsacchi, M. Caporali, M. Muccini, M. Peruzzini, M. Serrano-Ruiz, L. Calucci, M. A. Castriciano and S. Toffanin, ACS Appl. Mater. Interfaces, 2019, 11, 22637-22647.

17 E. Passaglia, F. Cicogna, G. Lorenzetti, S. Legnaioli, M. Caporali, M. Serrano-Ruiz, A. Ienco and M. Peruzzini, RSC Adv., 2016, 6, 53777-53783.

18 G. Abellán, V. Lloret, U. Mundloch, M. Marcia, C. Neiss, A. Görling, M. Varela, F. Hauke and A. Hirsch, Angew. Chem., Int. Ed., 2016, 55, 14557-14562.

19 S. Moschetto, M. Bolognesi, F. Prescimone, M. Brucale, A. Mezzi, L. Ortolani, M. Caporali, P. Pingue, M. SerranoRuiz, D. Pisignano, M. Peruzzini, L. Persano and S. Toffanin, ACS Appl. Nano Mater., 2021, 4, 3476-3485.

20 J. Plutnar, Z. Sofer and M. Pumera, ACS Nano, 2018, 12, 8390-8396.

21 V. V. Kulish, O. I. Malyi, C. Persson and P. Wu, Phys. Chem. Chem. Phys., 2014, 17, 992-1000.

22 T. Hu and J. Hong, J. Phys. Chem. C, 2015, 119, 8199-8207.

23 Y. Jing, X. Zhang and Z. Zhou, WIREs Comput. Mol. Sci., 2016, 6, 5-19.

24 P. Rastogi, S. Kumar, S. Bhowmick, A. Agarwal and Y. S. Chauhan, arXiv:1503.04296 [cond-mat].

25 L. Seixas, A. Carvalho and A. H. Castro Neto, Phys. Rev. B: Condens. Matter Mater. Phys., 2015, 91, 155138.

26 X. Sui, C. Si, B. Shao, X. Zou, J. Wu, B.-L. Gu and W. Duan, J. Phys. Chem. C, 2015, 119, 10059-10063.

27 H. Wang, S. Zhu, F. Fan, Z. Li and H. Wu, J. Magn. Magn. Mater., 2016, 401, 706-710.

28 M. Vanni, M. Serrano-Ruiz, F. Telesio, S. Heun, M. Banchelli, P. Matteini, A. M. Mio, G. Nicotra, C. Spinella, S. Caporali, A. Giaccherini, F. D'Acapito, M. Caporali and M. Peruzzini, Chem. Mater., 2019, 31, 5075-5080.

29 A. Ienco, G. Manca, M. Peruzzini and C. Mealli, Dalton Trans., 2018, 47, 17243-17256.

30 A. Ienco, M. Peruzzini and G. Manca, Dalton Trans., 2020, 49, 15072-15080.

31 C. J. An, Y. H. Kang, C. Lee and S. Y. Cho, Adv. Funct. Mater., 2018, 28, 1800532.

32 J. Hu, Z. Guo, P. E. Mcwilliams, J. E. Darges, D. L. Druffel, A. M. Moran and S. C. Warren, Nano Lett., 2016, 16, 74-79.

33 A. Yang, D. Wang, X. Wang, D. Zhang, N. Koratkar and M. Rong, Nano Today, 2018, 20, 13-32.

34 M. Caporali, M. Serrano-Ruiz, F. Telesio, S. Heun, G. Nicotra, C. Spinella and M. Peruzzini, Chem. Commun., 2017, 53, 10946-10949.

35 G. Qu, T. Xia, W. Zhou, X. Zhang, H. Zhang, L. Hu, J. Shi, X.-F. Yu and G. Jiang, Chem. Rev., 2020, 120, 2288-2346.

36 J. Wang, H. Zhang, X. Xiao, D. Liang, X. Liang, L. Mi, J. Wang and J. Liu, Acta Biomater., 2020, 107, 260-271.

37 D. Zhang, X. Lin, S. Lan, H. Sun, X. Wang, X. Liu, Y. Zhang and Y. Zeng, Part. Part. Syst. Charact., 2018, 35, 1800010.
38 D. Huang, Z. Zhuang, Z. Wang, S. Li, H. Zhong, Z. Liu, Z. Guo and W. Zhang, Appl. Surf. Sci., 2019, 497, 143825.

39 Q. Wu, M. Liang, S. Zhang, X. Liu and F. Wang, Nanoscale, 2018, 10, 10428-10435.

40 T. H. Lee, S. Y. Kim and H. W. Jang, Nanomaterials, 2016, 6, 194.

41 R. Xu, J. Yang, Y. Zhu, H. Yan, J. Pei, Y. W. Myint, S. Zhang and Y. Lu, Nanoscale, 2015, 8, 129-135.

42 G. J. Hutchings and M. Haruta, Appl. Catal., A, 2005, 291, 2-5.

43 D. C. Lim, I. Lopez-Salido and Y. D. Kim, Surf. Sci., 2005, 598, 96-103.

44 G. Barcaro and A. Fortunelli, New J. Phys., 2007, 9, 22-22.

45 D. Latha, P. Prabu, G. Gnanamoorthy, S. Munusamy, S. Sampurnam, C. Arulvasu and V. Narayanan, SN Appl. Sci., 2018, 1, 134.

46 P. Suchomel, L. Kvitek, R. Prucek, A. Panacek, A. Halder, S. Vajda and R. Zboril, Sci. Rep., 2018, 8, 4589.

47 S. Lange, P. Schmidt and T. Nilges, Inorg. Chem., 2007, 46, 4028-4035.

48 M. Bolognesi, M. Brucale, A. Lorenzoni, F. Prescimone, S. Moschetto, V. V. Korolkov, M. Baldoni, M. Serrano-Ruiz, M. Caporali, F. Mercuri, E. Besley, M. Muccini, M. Peruzzini, P. H. Beton and S. Toffanin, Nanoscale, 2019, 11, 17252-17261.

49 D. Nečas and P. Klapetek, Cent. Eur. J. Phys., 2012, 10, 181188.

50 R. Dovesi, V. R. Saunders, C. Roetti, R. Orlando, C. M. Zicovich-Wilson, F. Pascale, B. Civalleri, K. Doll, N. M. Harrison, I. J. Bush, P. D’Arco, M. Llunell, M. Causà, Y. Noël, L. Maschio, A. Erba, M. Rerat and S. Casassa, CRYSTAL17, CRYSTAL17 User's Manual, University of Torino, Torino, 2017.

51 A. D. Becke, J. Chem. Phys., 1993, 98, 5648-5652.

52 P. J. Hay and W. R. Wadt, J. Chem. Phys., 1985, 82, 299-310.

53 R. Weihrich and I. Anusca, Z. Anorg. Allg. Chem., 2006, 632, 335-342.

54 T. Zhang, Z. Cheng, Y. Wang, Z. Li, C. Wang, Y. Li and Y. Fang, Nano Lett., 2010, 10, 4738-4741.

55 A. Ciesielski, S. Haar, M. El Gemayel, H. Yang, J. Clough, G. Melinte, M. Gobbi, E. Orgiu, M. V. Nardi, G. Ligorio, V. Palermo, N. Koch, O. Ersen, C. Casiraghi and P. Samorì, Angew. Chem., Int. Ed., 2014, 126, 10523-10529.

56 S. Liu, N. Huo, S. Gan, Y. Li, Z. Wei, B. Huang, J. Liu, J. Li and H. Chen, J. Mater. Chem. C, 2015, 3, 10974-10980.

57 J. Lin, L. Liang, X. Ling, S. Zhang, N. Mao, N. Zhang, B. G. Sumpter, V. Meunier, L. Tong and J. Zhang, J. Am. Chem. Soc., 2015, 137, 15511-15517.

58 Y. Feng, J. Zhou, Y. Du, F. Miao, C.-G. Duan, B. Wang and X. Wan, J. Phys.: Condens. Matter, 2015, 27, 185302.

59 C. R. Ryder, J. D. Wood, S. A. Wells, Y. Yang, D. Jariwala, T. J. Marks, G. C. Schatz and M. C. Hersam, Nat. Chem., 2016, 8, 597-602.

60 H. Wu, X. Huang, M. Gao, X. Liao and B. Shi, Green Chem., 2011, 13, 651-658. 
61 V. Amendola, Phys. Chem. Chem. Phys., 2016, 18, 22302241.

62 G. T. Forcherio and D. K. Roper, Adv. Opt. Mater., 2016, 4, 1288-1294.

63 W. Luo, D. Y. Zemlyanov, C. A. Milligan, Y. Du, L. Yang, Y. Wu and P. D. Ye, Nanotechnology, 2016, 27, 434002.

64 S. Peters, S. Peredkov, M. Neeb, W. Eberhardt and M. AlHada, Surf. Sci., 2013, 608, 129-134.
65 S. P. Koenig, R. A. Doganov, L. Seixas, A. Carvalho, J. Y. Tan, K. Watanabe, T. Taniguchi, N. Yakovlev, A. H. Castro Neto and B. Özyilmaz, Nano Lett., 2016, 16, 2145-2151.

66 J. Xu, X. Qiao, Y. Wang, Q. Sheng, T. Yue, J. Zheng and M. Zhou, Microchim. Acta, 2019, 186, 238.

67 H. Zhao, W. Zhang, Z. Liu, D. Huang, W. Zhang, B. Ye, G. Hu, H. Zhong, Z. Zhuang and Z. Guo, Nanophotonics, 2018, 7, 1651-1662. 